(Aus der Kgl. Universitäts-Poliklinik für innere Kranke. Geh.-Rat Prof. Dr. Senator.)

\title{
Beitrag zur Kenntnis vom Einfluss der Röntgenstrahlen auf die Geschlechtsorgane.
}

\author{
Von
}

\section{Heinmich Gerhartz.}

Das Studium des Einflusses, den die Röntgenstrahlen auf die Generationsorgane ausüben, kann jach zwei Seiten hin geführt werden. Einmal ist zu untersuchen die Wirkung auf die spezifischen Geschlechtszellen, das andere Mal die auf das innere Sekret der Geschlechtsdrüse. Was bisher Positives zutage gefördert wurde, beschränkt sich fast nur auf das erstere Kapitel. Hier liegt das eigentliche Fundament unserer heutigen Anschauungen über die biologische Bedeutung der Röntgenstrahlen.

Es schien mir am fruchtbarsten für die weitere Aufklärung, vergleichend-physiologisch vorzugehen und mich dabei, weil dieser Weg die klarsten Aufschlüsse zu geben schien, an die Methode meines früheren Lehrers M. Nussbau m zu halten. Hiernach ergab sich eine Versuchsanordnung an periodisch brünstigen Tieren in der Art, dass Landfrösche (Rana fusea o und o) vor der Zeit der im Zyklus erfolgenden Ausbildung der Geschlechtsdrüsen täglich intensiv der Wirkung der Röntgenstrahlen ausgesetzt wurden.

Am 25. Juli 1907 wurde mit den Bestrahlungen begonnen. Um diese Zeit ist die innersekretorische Tätigkeit der Geschlechtsdrüse des Männchens noch nicht in der Ausbildung der Daumenschwielen zum sichtbaren Ausdruck gekommen. Da diese Brunstattribute mit dem Monat September hypertrophieren und in der Mitte dieses Monates schon eine beträchtliche Grösse erreicht haben ${ }^{1}$ ), ferner die

1) M. Nussbaum, Einfluss des Hodensekrets auf die Entwicklung der Brunstorgane des Landfrosches. Sitzungsber. der Niederrh. Gesellsch. f. Naturu. Heilkunde 1905 S. 44-46. Bonn 1906. 
Regeneration der Samenblasen um diese Zeit in vollem Gange ist, wurde der Versuch vom 3. bis 19. September abgebrochen.

Bei der Bestrahlung waren die Tiere von der Wasserküblröhre $27 \mathrm{~cm}$ entfernt; es wurde mit einem $50 \mathrm{~cm}$-Induktor und $\mathrm{Hg}$-Unterbrecher gearbeitet. Die Dosis betrug 20-30 MA (Ruhmer'sche Messröhre), die Dauer 10 Minuten.

Die Ergebnisse sind in den nachstehenden Tabellen I und II, (S. 570) die ohne weiteres verständlich sind, zusammengestellt. Die Zahlen sind durch Mikrometermessungen gewonnen. Unter proximaler Schwiele ist die des Pollexrudimentes, unter distaler die daneben am Metacarpale des zweiten Gliedes liegende verstanden.

Die Tiere sind während der Dauer des Versuches schlecht ernährt worden. Trotzdem aber sind die Geschlechtsdrüsen, Daumenschwielen und Samenblasen, wie aus den Daten der beiden Tabellen hervorgeht, der Norm entsprechend gewachsen. In den Hoden wurde keinerlei degenerative Veränderung gefunden.

Bei drei weiteren, weiblichen Tieren waren die Ovarien ebenfalls zur vollen Entwicklung gekommen. Nur ein Tier hatte, wie schon die Besichtigung mit blossem Auge ergab, einen schlechter entwickelten Eierstock. Herr Prof. M. Nussbaum hat die Güte gehabt, auch die histologischen Präparate dieses Organs, wie die übrigen Schnitte, einer Durchsicht zu unterziehen. Es wurde dabei ein Befund erhoben, der sich mit den sonstigen Anzeichen des Hungers deckt. Das darf bei der starken Gewichtsabnahme des betreffenden Exemplars $(8,0 \mathrm{~g}$ in 6 Wochen) nicht überraschen.

Diese Befunde, die infolge der kombinierenden Einwirkung des Hungers a fortiori beweiskräftig sind, zeigen, dass ein schädigender Einfluss der Röntgenstrahlen auf das innere Sekret der Hoden und auf die in der Ausbildung begriffenen Geschlechtsdrüsen bei der angewandten recht hohen Bestrahlungsdosis nicht statthat. Sie stehen in vollem Gegensatz zu den Ergebnissen anderer Forscher, die an höheren Tieren experimentiert haben. Um diese Divergenz aufzuklären und meine Befunde zu kontrollieren und zu vervollständigen, wurden neue Versuche in der Weise angestellt, dass nun drei männliche Ranae fuscae, deren Geschlechtsorgane voll entwickelt waren, und gleichzeitig mit ihnen zwei Kaninchen bestrahlt wurden.

Die Tiere wurden zuerst am 18. Januar 1909. den Röntgenstrahlen ausgesetzt, dann weiter täglich 10-15 Minuten lang mit im Mittel 15-20 MA des $\mathrm{Ruhmer}$ 'schen Milliampèremeters: in $20 \mathrm{~cm}$ 
Heinrich Gerhartz:
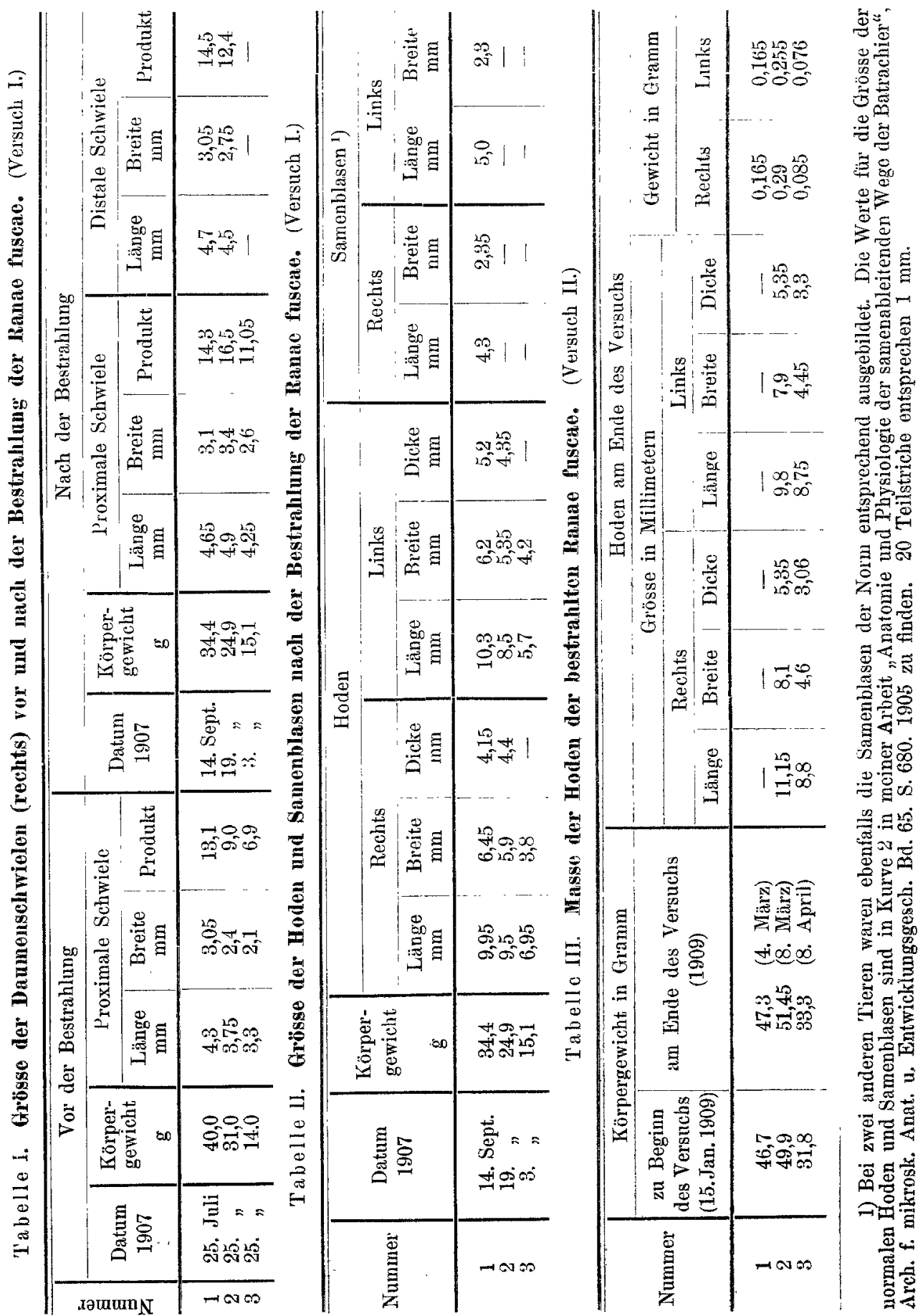
Röhrenabstand, unter sonst gleichen Bedingungen wie oben, bis zum “ 4. März 1909, also bis kurz vor dem Laichtermin der Frösche, bestrahlt. Sie wurden regelmässig mit Froschfleisch gut gefüttert, so dass beim Abschluss des Versuches das Körpergewicht etwas gestiegen war.

Die genauen Daten und die Grösse der Hoden sind in Tabelle III (S. 570) notiert.

Diese Tabelle zeigt, dass die Grösse der Hoden nach der Bestrahlung im allgemeinen der Norm entsprach.

Die histologische Untersuchung der Präparate ergab, dass die Spermatozoen sehr reichlich vorhanden und gut ausgebildet waren. Die Zahl der Spermatogonien batte bei allen Tieren, namentlich bei Tier Nr. 2, wo sie nur noch äusserst spärlich waren, abgenommen. Neben woblerhaltenen Spermatogonien wurden typische Degenerationsformen der Kerne gefunden. Die Kerne des interstitiellen Gewebes waren nicht geschädigt worden. Mitotische Vermehrung wurde nicht beobachtet. Die Bestrahlung hatte bei den Fröschen augenscheinlich zu einer Vernichtung von Spermatogonien geführt, die an Ausdehnung weit zurückblieb hinter der Schädigung, die die Geschlechtsdrüse und ibr Produkt bei den reifen höberen Tieren ${ }^{1}$ ) (hier beim Kaninchen) unter den gleichen Umständen erleiden.

Aus den Befunden an Tier 3 ging hervor, dass diese Schädigung nicht irreparabel war. Die Zahl der Spermatogonien hatte 4 Wochen nach dem Aufhören der Bestrahlung wieder erheblich zugenommen.

1) Siehe die Literatur bei K. Fr. Hoffmann, Über den Einfluss der Röntgenstrahlen auf den Kaninchenhoden. Diss. Bonn 1908. - Vgl. ferner Jul. Tandler und Siegfr. Grosz, Untersuchungen an Skopzen. Wien. klin. Wochenschr. Bd. 21. S. 277-282. 1908. 